\title{
Critical Behavior of a Supersymmetric Extension of the Ginzburg-Landau Model
}

\author{
Grigoris Panotopoulos \\ Departament de Fisica Teorica, Universitat de Valencia, Burjassot, Spain; Instituto de Fisica Corpuscular (IFIC), \\ Institutos de Paterna, Universitat de Valencia-CSIC, Valencia, Spain \\ E-mail: Grigoris.Panotopoulos@uv.es \\ Received January 31, 2011; revised February 26, 2011; accepted March 18, 2011
}

\begin{abstract}
We make a connection between quantum phase transitions in condensed matter systems, and supersymmetric gauge theories that are of interest in the particle physics literature. In particular, we point out interesting effects of the supersymmetric quantum electrodynamics upon the critical behavior of the Ginzburg-Landau model. It is shown that supersymmetry fixes the critical exponents, as well as the Landau-Ginzburg parameter, and that the model resides in the type II regime of superconductivity.
\end{abstract}

Keywords: Superconductivity, Critical Exponents, Supersymmetry

\section{Introduction}

A very well studied model in the condensed matter literature is the Ginzburg-Landau (GL) model [1], described by the lagrangian of an Abelian Higgs model

$$
\mathcal{L}=\left|D_{\mu} \phi\right|^{2}+m^{2}|\phi|^{2}+\lambda|\phi|^{4}+\frac{1}{4} F_{\mu v}^{2}
$$

where $\phi$ is a complex scalar field charged under the abelian gauge field $A_{\mu}$, with the gauge covariant derivative and field strength

$$
\begin{gathered}
D_{\mu}=\partial_{\mu}-i e A_{\mu} \\
F_{\mu \nu}=\partial_{\mu} A_{\nu}-\partial_{\nu} A_{\mu}
\end{gathered}
$$

When $m^{2}>0$, the gauge symmetry is exact, and the model describes a massive complex scalar particle that interacts with a massless photon. The electric potential between these scalars has the usual Coulomb form, and therefore this is referred to as the Coulomb phase. On the other hand, when $m^{2}<0$ the gauge symmetry is spontaneously broken, and in this Higgs phase the model describes a massive gauge boson and a massive real scalar field. The nature of the transition between the Higgs and Coulomb phase has been of great interest to the condensed matter community.

The critical fluctuations in the Ginzburg-Landau model of superconductors were studied in [2], while the fixed point structure for the GL model was presented in [3].
Furthermore, in previous works the authors have investigated models in which massless Dirac fermions are coupled to the Ginzburg-Landau model [4]. The presence of the Dirac fermions is justified by the fact that effective microscopic models of strongly correlated electrons usualy contain them [5]. The critical exponents can be computed as a function of the number $N_{F}$ of the fermions, and for increasing $N_{F}$ the models is driven into the type II regime of superconductivity. In particular, for the minimum allowed value of the fermion number, $N_{F}=4$, both values of the $\kappa$ parameter, corresponding to the ' $\mathrm{T}$ ' fixed point and the ' $\mathrm{SC}$ ' fixed point, are found to be above the mean-field GL value $1 / \sqrt{2}$, in contrast to the theoretical [6] and the Monte Carlo numbers [7] in the GL model. In this article we point out that the generalization of the Ginzburg-Landau model to a supersymmetric one necessarily introduces fermions both in the matter and gauge supermultiplets, and that the restrictions imposed by the symmetries of the model unambigiously determine the critical exponents and the Landau-Ginzburg parameter, which is found to be in the type II regime of superconductivity.

Finally, we remind the reader that a) all exactly solvable models show that not all of the critical exponents are independent. In fact they satisfy certain scaling laws, supported by all the experimental and numerical results, and it can be shown that there are only two independent critical exponents. If we take them to be $\eta$ and $v$, the rest of the critical exponents are given by $[8,9]$ : 


$$
\begin{gathered}
\alpha=2-v D \\
\beta=\frac{v}{2}(D-2+\eta) \\
\gamma=v(2-\eta) \\
\delta=\frac{D+2-\eta}{D-2+\eta}
\end{gathered}
$$

where $D$ is the dimension of the system, and b) in the Landau-Ginzburg theory there are two fundamental length scales, namely the penetration length $\lambda$ and the coherence length $\xi_{0}$. The Landau-Ginzburg parameter $\kappa$ is defined as follows

$$
\kappa \equiv \frac{\lambda}{\xi_{0}}
$$

and it can be shown that $\kappa<1 / \sqrt{2}$ corresponds to type I superconductors, while $\kappa>1 / \sqrt{2}$ corresponds to type II superconductors.

\section{The Supersymmetric Model and Critical Exponents}

Supersymmetric Quantum Electrodynamics (SQED) is an abelian gauge theory with the following field content [10]:

1) One vector multiplet $\left(A^{\mu}, \lambda^{\alpha}, \bar{\lambda} \dot{\alpha}\right)$ consisting of the photon and the photino (in the so-called Wess-Zumino gauge), described by a vector and a Majorana spinor field.

2) Two chiral multiplets $\left(\psi_{L}^{\alpha}, \phi_{L}\right)$ and $\left(\psi_{R}^{\alpha}, \phi_{R}\right)$ with charges $Q_{L}=-1, Q_{R}=+1$, each consisting of one Weyl spinor and one scalar field, constituting the left- and right-handed electron and selectron, the matter fields.

The electron Dirac spinor and the photino Majorana spinor are given by

$$
\Psi=\left(\begin{array}{l}
\psi_{L \alpha} \\
\bar{\psi}_{R}^{\dot{\alpha}}
\end{array}\right), \quad \tilde{\gamma}=\left(\begin{array}{c}
-i \lambda_{\alpha} \\
i \bar{\lambda}^{\dot{\alpha}}
\end{array}\right) .
$$

The SQED Lagrangian contains kinetic, minimal coupling and mass terms and in addition, due to the supersymmetry, coupling terms to the photino and quartic terms in the selectron fields:

$$
\begin{aligned}
& L_{\mathrm{SQED}}=-\frac{1}{4} F_{\mu \nu} F^{\mu v}+\frac{1}{2} \overline{\tilde{\gamma}} i \gamma^{\mu} \partial_{\mu} \tilde{\gamma} \\
& +\left|D_{\mu} \phi_{L}\right|^{2}+\left|D_{\mu} \phi_{R}^{\dagger}\right|^{2}+\bar{\Psi} i \gamma^{\mu} D_{\mu} \Psi \\
& -\sqrt{2} e Q_{L}\left(\bar{\Psi} P_{R} \tilde{\gamma} \phi_{L}-\bar{\Psi} P_{L} \tilde{\gamma} \phi_{R}^{\dagger}+\phi_{L}^{\dagger} \overline{\tilde{\gamma}} P_{L} \Psi-\phi_{R} \overline{\tilde{\gamma}} P_{R} \Psi\right) \\
& -\frac{1}{2}\left(e Q_{L}\left|\phi_{L}\right|^{2}+e Q_{R}\left|\phi_{R}\right|^{2}\right)^{2}-m \bar{\Psi} \Psi-m^{2}\left(\left|\phi_{L}\right|^{2}+\left|\phi_{R}\right|^{2}\right)
\end{aligned}
$$

with the gauge covariant derivative and field strength

$$
\begin{aligned}
& D_{\mu}=\partial_{\mu}+i e Q A_{\mu}, \\
& F_{\mu \nu}=\partial_{\mu} A_{\nu}-\partial_{\nu} A_{\mu} .
\end{aligned}
$$

It must be noted that the model with just one chiral supermultiplet is anomalous, while the inclusion of a second chiral supermultiplet with opposite electric charge renders the model anomaly-free, since in this case $\operatorname{Tr} Q=0$. The two Weyl spinors combine to form the Dirac spinor of the usual spinor electrodynamics in the standard fourcomponent formalism.

The model contains both bosons and fermions, with equal masses and degrees of freedom within each multiplet. The form of the interactions, as well as the values of the couplings, are completely determined by the symmetries. It is interesting that there is just one coupling constant, namely the electric charge $e$. We have the usual types of interaction that one encounters in the usual field theory, namely quartic interaction for the scalars, Yukawa coupling, and the gauge (electromagnetic) interaction. We thus know that the theory is renormalizable. In fact, here we have just a wave function renormalization both for the vector and the chiral multiplets due to supersymmetry [11], and furthermore the beta function for the electric charge is determined by the photon self-energy and wave-function renormalization due to gauge invariance [12].

The investigation regarding the critical behavior is according to the following program: a) Perform a one-loop analysis to compute the relevant counterterms that eliminate the unwanted divergencies, b) determine the betafunction for the electric charge $\beta(e)$, as well as the anomalous dimensions for the scalars $\gamma_{m}, \gamma_{\phi}$, c) find the fixed points from the condition $\beta\left(e_{*}\right)=0$, and finally $\mathrm{d}$ ) compute the critical exponents $\eta, v$ using the wellknown formulas $[4,13]$

$$
\begin{gathered}
\eta=2 \gamma_{\phi^{*}} \\
v=\frac{1}{2\left(1-\gamma_{m^{*}}\right)}
\end{gathered}
$$

where the anomalous dimensions, as well as the beta function are given by [14]

$$
\begin{gathered}
\beta(\alpha)=\mu \frac{\partial \alpha}{\partial \mu} \\
\gamma_{\phi}=\frac{1}{2} \mu \frac{\partial \ln Z_{\phi}}{\partial \mu} \\
\gamma_{m}=\frac{\mu}{m} \frac{\partial m}{\partial \mu}
\end{gathered}
$$

with $\mu$ the renormalization mass scale, and $\alpha=e^{2} / 4 \pi$ the fine-structure constant. Note that our definitions for the anomalous dimensions are slightly different than $[4,13]$. 
We start from the photon self energy, that will allows to compute the electric charge beta function $\beta(\alpha)$. The relevant loop-diagrams are shown in Figure 1. We have the same diagrams as in the usual spinor and scalar electrodynamics. The electric charge beta function has a contribution from a Dirac spinor and a contribution from two complex scalars. At one loop and using dimensional regularization [15] (the space-time dimension $4 \rightarrow$ $D=4-\varepsilon$, then take the limit $\varepsilon \rightarrow 0$ and isolate the divergent part $\sim 1 / \varepsilon$ ) one obtains the result

$$
\beta(\alpha, \varepsilon)=-\varepsilon \alpha+\frac{\alpha^{2}}{\pi}
$$

Next we turn to the scalar field self-energy. The relevant diagrams can be shown in Figure 2. We have the three usual diagrams from scalar electrodynamics, plus a new one with the Yukawa coupling with the Dirac electron and the photino Majorana fermion. For the scalar field wave-function renormalization we find the result (in the Lorentz gauge)

$$
Z_{\phi}=1+\frac{5 e^{2}}{8 \pi^{2} \varepsilon}
$$

Now it is a straightforward algebraic task to compute the anomalous dimensions and then the critical exponents. We thus obtain our final results (for $D=3$ or $\varepsilon=1$ )

$$
\eta=-2.5
$$

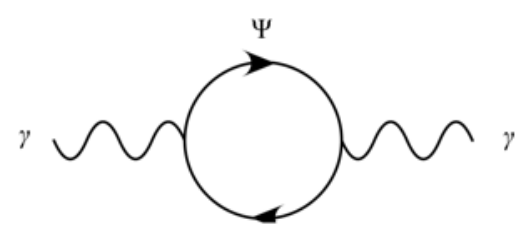

(a)

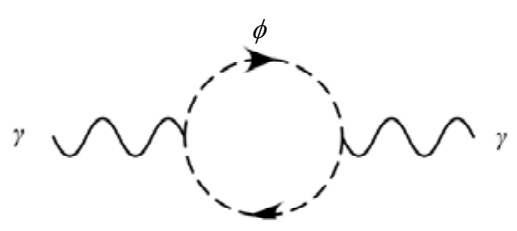

(b)

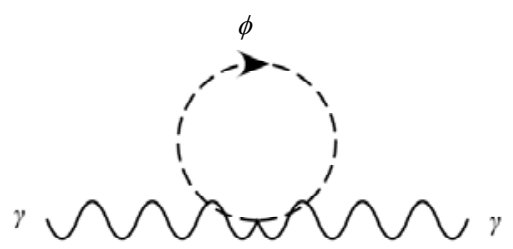

(c)

Figure 1. Feynman diagrams for the photon self-energy with the usual spinor and scalar electrodynamics interaction vertices.

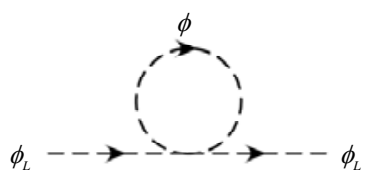

(a)

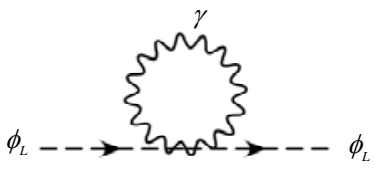

(c)

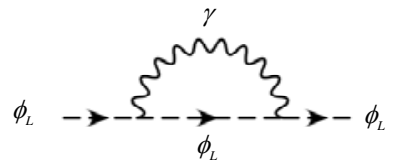

(b)

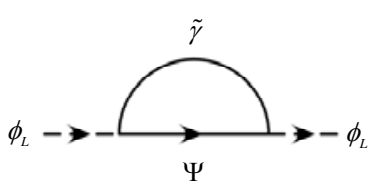

(d)
Figure 2. Feynman diagrams for the scalar self-energy with (a) the quartic, (b) the single photon, (c) the two-photon, and (d) the Yukawa interaction vertices.

$$
v=\frac{1}{6} \simeq 0.17
$$

Our results for $\beta(e)$ and $\gamma_{\phi}$ agree with the corresponding formulas of [16] at one loop. In [4] there are $N_{F}$ massless fermions, and two coupling constants with two different beta functions. The authors in [4] have found two fixed points (tricritical and superconducting), and that the number of massless fermions must be at least four. The $\eta$ critical exponent is always negative, while the $v$ critical exponent is always positive, and for both exponents the absolute value is a number around 0.5 when $N_{F}$ is small. In our supersymmetric version of the model, there is just one massive fermion, since supersymmetry requires that there are equal number of fermionic and bosonic degrees of freedom, and with the same masses. There is only one coupling constant, namely the electric charge $e$, and thus just one beta function, and a single infrared stable fixed point. Despite this, there is also here a quartic self-interaction potential for the scalar field, where the coupling is fixed by supersymmetry, and it is given in terms of the electric charge. Furthermore, we find also a negative $\eta$ critical exponent and a positive $v$ exponent, with values not too different from the ones obtained in [4] for small $N_{F}$.

\section{Supersymmetry Breaking and the $\kappa$ Parameter}

So far we have not seen any superpartners yet, and thus supersymmetry must be broken. In this section we shall discuss spontaneous breaking of supersymmetry, following [17], within the framework of the Fayet-Iliopoulos mechanism [18]. In an abelian $U(1)$ supersymmetric gauge theory an extra term is allowed by the symmetries, the so called Fayet-Iliopoulos term, $\xi D$, where $D$ is the auxiliary field in the vector supermultiplet, and $\xi$ is 
a new parameter with mass dimension two. If $F_{1}, F_{2}, D$ are the auxiliary fields in the off-shell formulation of the supersymmetric theory, the scalar potential is given by

$$
\mathcal{V}=\frac{1}{2} D^{2}+F_{1} F_{1}^{*}+F_{2} F_{2}^{*}
$$

and the auxiliary fields satisfy the following equations of motion

$$
\begin{gathered}
F_{1}+m A_{2}^{*}=0 \\
F_{2}+m A_{1}^{*}=0 \\
D+\xi+\frac{e}{2}\left(A_{1} A_{1}^{*}-A_{2} A_{2}^{*}\right)=0
\end{gathered}
$$

where now the scalar fields are denoted by $A_{1}, A_{2}$ instead of $\phi_{L}, \phi_{R}$. Supersymmetry is broken since there is no solution that leaves $\mathcal{V}=0$. Upon substitution the scalar potential takes the form

$$
\begin{aligned}
\mathcal{V} & =\frac{\xi}{2}+\left(m^{2}+\frac{e \kappa}{2}\right) A_{1} A_{1}^{*}+\left(m^{2}-\frac{e \xi}{2}\right) A_{2} A_{2}^{*} \\
& +\frac{1}{8} e^{2}\left(A_{1} A_{1}^{*}-A_{2} A_{2}^{*}\right)^{2}
\end{aligned}
$$

We can see that there are two possibilities, namely that $m^{2}>e \xi / 2$ or $m^{2}<e \xi / 2$. In the first case the $A_{1}=$ $0=A_{2}$ minimizes the potential, the form of which is shown in Figure 3(a). The supersymmetry is spontabneously broken but the gauge symmetry is exact. The theory describes two complex scalar fields with masses $m^{2}+\frac{e \xi}{2}$ and $m^{2}-\frac{e \xi}{2}$. The rest of the fields, namely the photon $\gamma$, the photino $\lambda$, and the two fermions $\psi_{1}, \psi_{2}$ retain their masses. In particular, the photino is the massless goldstino. In the second case the $A_{1}=0=A_{2}$ no longer minimizes the potential, the form of which is shown in Figure 3(b). This time both the supersymmetry and the gauge symmetry are broken simultaneously. The minimum corresponds to $A_{1}=0, A_{2}=v$, where the vacuum expectation value $v$ is determined from

$$
\frac{e^{2} v^{2}}{4}+\left(m^{2}-\frac{e \xi}{2}\right)=0
$$

This model describes a vector field and a scalar field of mass $\sqrt{e \xi / 2}$, a complex scalar field with mass $\sqrt{2 m^{2}}$, a massless goldstino, and two spinor fields with mass $\sqrt{m^{2}+\frac{e \xi}{2}}$. The Landau-Ginzburg parameter therefore is easily computed to be

$$
\kappa=\frac{m_{s}}{m_{v}}=1 \simeq \frac{1.41}{\sqrt{2}}
$$

which is larger than $1 / \sqrt{2}$, and we thus have a type II

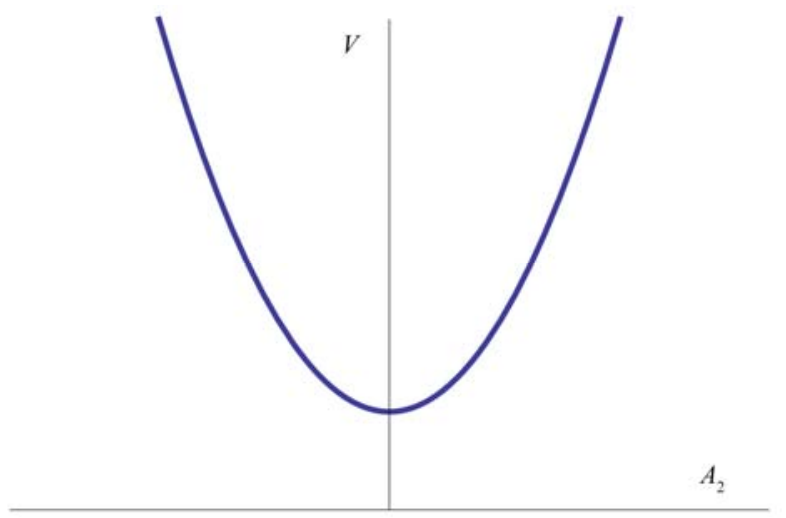

(a)

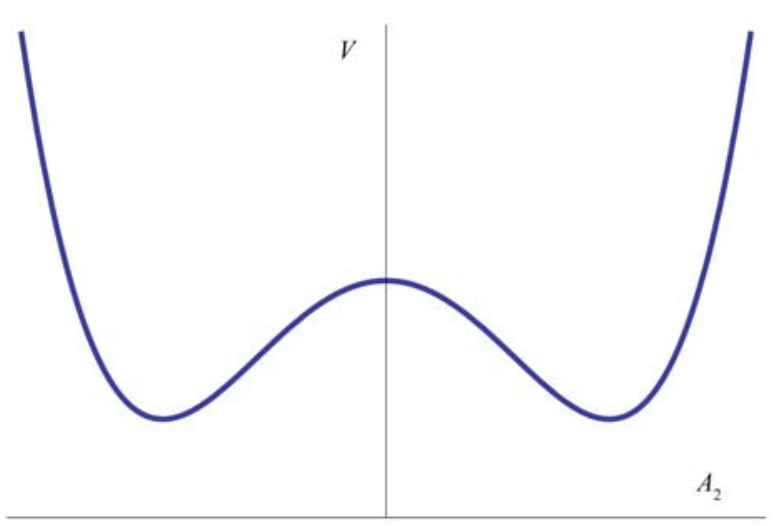

(b)

Figure 3. (a) The scalar potential versus $A_{2}$ in the $m^{2}>e \kappa / 2$ case (in arbitrary units). Supersymmetry is spontaneously broken, but the $U(1)$ gauge symmetry is exact. (b) As in (a) but in the $m^{2}<e \kappa / 2$ case. Here, both supersymmetry and $U(1)$ gauge symmetry are spontaneously broken.

superconductor. It is interesting to see again that our value of the Ginzburg parameter is comparable to the value obtained in [4] at the superconducting fixed point and for $N_{F}=4$. Therefore, we conclude that supersymmetry provides the kind of lagrangian studied in [4], and that the values of the Ginzburg parameter and of the critical exponents are similar to the ones obtained in [4], without a second coupling constant $\lambda$ for the scalar quartic self-interaction, and without many fermions.

\section{Conclusions}

We have proposed and analyzed a supersymmetric extension of the Landau-Ginzburg theory, which is essentially the supersymmetric version of quantum electrodynamics. The model describes the interaction of a Dirac fermion and two complex scalar fields with the photon and its superpartner, the photino, which is a Majorana 
fermion. All the couplings in the model are given in terms of the electric charge. It is interesting that there is a quartic self-interaction coupling for the scalar fields even in the absence of a coupling $\lambda$. Within the one-loop renormalization program we give the expression for the wave-function renormalization, and according to the standard prescription we compute the critical exponents $\eta, v$ from the beta function and the anomalous dimensions. Finally, we have discussed spontaneous supersymmetry breaking a la Fayet-Iliopoulos mechanism. There is a case in which both supersymmetry and gauge symmetry can be broken at the same time. The photon acquires a non-vanishing mass, and the Landau-Ginzburg parameter is computed. We find that its value corresponds to type II superconductors. Our values of the Ginzburg parameter and of the critical exponents are similar to the ones obtained in [4], without many fermions and without the introduction of a second coupling constant for the scalar quartic self-interaction.

\section{Acknowledgments}

The author acknowledges financial support from FPA 2008-02878 and Generalitat Valenciana under the grant PROMETEO/2008/004.

\section{References}

[1] V. L. Ginzburg and L. D. Landau, "On the Theory of Superconductivity," Zhurnal Eksperimental'noi i Teoreticheskoi Fiziki, Vol. 20, 1950, pp. 1064-1082.

[2] B. I. Halperin, T. C. Lubensky and S. K. Ma, "FirstOrder Phase Transitions in Superconductors and Smectic-A Liquid Crystals," Physical Review Letters, Vol. 32, 1974, pp. 292-295. J. H. Chen, T. C. Lubensky and D. R. Nelson, "Crossover near Fluctuation-Induced First-Order Phase Transitions in Superconductors," Physical Review B, Vol. 17, No. 11, 1978, pp. 4274-4286. doi:10.1103/PhysRevB.17.4274

[3] I. D. Lawrie, "On the Phase Transitions in Abelian Higgs Models," Nuclear Physics B, Vol. 200, No. 1, 1982, pp. 1-19. doi:10.1016/0550-3213(82)90055-4

I. F. Herbut and Z. Tesanovic, "Critical Fluctuations in Superconductors and the Magnetic Field Penetration Depth," Physical Review Letters, Vol. 76, No. 24, 1996, pp. 4588-4591. doi:10.1103/PhysRevLett.76.4588

[4] H. Kleinert and F. S. Nogueira, "Critical Behavior of the Ginzburg-Landau Model Coupled to Massless Dirac Fermions," Physical Review B, Vol. 66, No. 1, 2002, p. 012504. doi:10.1103/PhysRevB.66.012504

[5] J. B. Marston, "U(1) Gauge Theory of the Heisenberg Antiferromagnet," Physical Review Letters, Vol. 61, No. 17, 1988, pp. 1914-1917. doi:10.1103/PhysRevLett.61.1914
J. B. Marston and I. Affleck, "Large-n Limit of the Hubbard-Heisenberg Model," Physical Review B, Vol. 39, No. 16, 1989, pp. 11538-11558.

doi:10.1103/PhysRevB.39.11538

D. H. Kim and P. A. Lee, "Theory of Spin Excitations in Undoped and Underdoped Cuprates," Annals of Physics, Vol. 272, No. 1, 1999, pp. 130-164.

doi:10.1006/aphy.1998.5888

[6] H. Kleinert, "Disorder Version of the Abelian Higgs Model and the Order of the Superconductive Phase Transition," Lettere Al Nuovo Cimento, Vol. 35, No. 13, 1982, pp. 405-412. doi:10.1007/BF02754760

[7] S. Mo, J. Hove and A. Sudbo, "Order of the Metal-to-Superconductor Transition," Physical Review B, Vol. 65, No. 10, 2002, p. 104501. doi:10.1103/PhysRevB.65.104501

[8] M. Le Bellac, "Quantum and Statistical Field Theory," Oxford University Press, Oxford, 1992.

[9] H. Kleinert and V. Schulte-Frohlinde, "Critical Phenomena in $\Phi^{4}$-Theory," World Scientific, Singapore, 2001. http://www.physik.fu-berlin.de/kleinert/b8

[10] W. Hollik, E. Kraus and D. Stockinger, "Renormalization and Symmetry Conditions in Supersymmetric QED," European Physical Journal C, Vol. 11, No. 2, 1999, pp. 365-381. doi:10.1007/s100529900216

[11] S. Ferrara and O. Piguet, "Perturbation Theory and Renormalization of Supersymmetric Yang-Mills Theories," Nuclear Physics B, Vol. 93, No. 2, 1975, pp. 261-302. doi:10.1016/0550-3213(75)90573-8

[12] J. D. Bjorken and S. D. Drell, "Relativistic Quantum Mechanics," McGraw-Hill, New York, 1998.

[13] F. S. Nogueira and H. Kleinert, "Field Theoretical Approaches to the Superconducting Phase Transition," The Smithsonian/NASA Astrophysics Data System.

[14] M. E. Peskin and D. V. Schroeder, "An Introduction to Quantum Field Theory (Frontiers in Physics)," Westview Press, New York, 1995.

M. Srednicki, "Quantum Field Theory," Cambridge University Press, Cambridge, 2007.

[15] G. 't Hooft and M. J. G. Veltman, "Regularization and Renormalization of Gauge Fields," Nuclear Physics B, Vol. 44, No. 1, 1972, pp. 189-213. doi:10.1016/0550-3213(72)90279-9

[16] M. E. Machacek and M. T. Vaughn, "Two-Loop Renormalization Group Equations in a General Quantum Field Theory: (I). Wave Function Renormalization," Nuclear Physics B, Vol. 222, No. 1, 1983, pp. 83-103. doi:10.1016/0550-3213(83)90610-7

[17] J. Wess and J. Bagger, "Supersymmetry and Supergravity," Princeton University Press, New Jersey, 1992.

[18] P. Fayet and J. Iliopoulos, "Spontaneously Broken Supergauge Symmetries and Goldstone Spinors," Physics Letters B, Vol. 51, No. 5, 1974, pp. 461-464. doi:10.1016/0370-2693(74)90310-4 